\title{
Two-dimensional orthoglide mechanism for revealing areflexive human arm mechanical properties
}

\author{
Hannes Höppner ${ }^{1}$, Markus Grebenstein ${ }^{1}$, and Patrick van der Smagt ${ }^{2,3}$
}

\begin{abstract}
The most accurate and dependable approach to the in-vivo identification of human limb stiffness is by position perturbation. Moving the limb over a small distance and measuring the effective force gives, when states are steady, direct information about said stiffness. However, existing manipulandi are comparatively slow and/or not very stiff, such that a lumped stiffness is measured. This lumped stiffness includes the limb response during or after reflexes influenced by both, the passive musculotendon and active neuronal component.

As this approach usually leads to inconsistencies between the data and the stiffness model, we argue in favour of fast, pre-reflex impedance measurementsi.e., completing the perturbation movement and collecting the data before effects of spinal reflexes or even from the motor cortex can influence the measurements. To obtain such fast planar movements, we constructed a dedicated orthoglide robot while focusing on a lightweight and stiff design. Our subject study of a force task with this device lead to very clean data with always positive definite Cartesian stiffness matrices. By representing them as ellipses, we found them to be substantially bigger in comparison to standard literature which we address to a larger number of recruited motor units. While ellipses orientation and the length of their main axis increased, the shape decreased with the exerted force. The device will be used to derive design criteria for variable-stiffness robots, and to investigate the relation between muscular activity and areflexive joint stiffness for teleoperational approaches.
\end{abstract}

\section{INTRODUCTION}

With the increasing dynamic behaviour of robotic systems, the question of how to handle unknown impact situations safely has raised a lot of interest in the past decade. Consequently, compliant robotic systems are an active field in robotics research. One of the pioneering systems is the LWR-III [1] with an active compliancecontrolled behaviour in 7 degrees of freedom.

Compliance in human limbs is caused by both reflexive and areflexive components. The reflexive (active) components are caused by fast, spinal, reflexive feedback loops with delays in the order of $25 \mathrm{~ms}$ (short latency reflex) plus $\sim 20 \mathrm{~ms}[2]$ (onset of muscle force after muscular activation). These relatively long delays indicate a

\footnotetext{
${ }^{1}$ These authors are with the Institute of Robotics and Mechatronics, German Aerospace Center (DLR), D-82234 Oberpfaffenhofen, Germany \{hannes.hoeppner, markus.grebenstein\}@dlr.de

${ }^{2}$ Patrick van der Smagt is with the Department for Informatics, Technische Universität München, Germany

${ }^{3}$ Patrick van der Smagt also is with fortiss, Associate Institute of the Technische Universität München, Germany
}

rather limited dynamic behaviour [3], hence the system is completed with an areflexive component caused by the pure mechanical properties of the musculotendon system, consisting of passive (tissue) and intrinsic properties (i.e. the cross-bridges). Different levels of isotonic muscular activation preloads the related muscles correspondingly, allowing for a different present of reflexive and areflexive responses.

This dichotomy of compliance is mimicked in robotic systems by further adding nonlinear, pretensionable, springs, in a mechanism known as variable-stiffness actuation (VSA) [4], [5], [6]. However, defining mechanical meaningful parameters for this passive compliance in robotics is still heuristically solved, as no general rules of stiffness variation have been devised. E.g., it is unknown to which extent humans can decouple an applied force from stiffness using the strategy of cocontraction, which might be used as an inspiration for designing forcestiffness characteristics of variable-stiffness robots.

Planar human arm motor studies are typically performed with a specific type of manipulandum: an asymmetric 5R parallel mechanism design [7], [8], [9], [10], [11]. Competing designs include the $5 \mathrm{R}$ symmetric parallel mechanism design [12], [13], the $2 \mathrm{P}$ cable-driven approach [14], and a compact cam disc-based device with one active degree of freedom [15].

Different techniques to investigate overall compliance in humans limbs have been developed. The most effective ones are steady-state perturbations which were introduced by Mussa-Ivaldi et al. in 1985 [7] and which are used to measure stiffness only. The limb is displaced by short positional perturbations and the reaction forces in two static positions are measured. Assuming that influences of velocity and acceleration are negligible, stiffness will be the main contribution to the measured response. However, since the manipulandum has a limited dynamic performance, the second static position was only reached after approximately $550 \mathrm{~ms}$ and the last data point was taken $1000 \mathrm{~ms}$ after the perturbation started. This shows the main drawback of these existing step perturbation methods: Since the stiffness estimates uses time windows substantially longer than the stretch, spinal, and longlatency reflex loops, and even longer than the onset of voluntary active response - which occurs within 300 to $500 \mathrm{~ms}$ [16] - subjects are usually instructed to not voluntarily intervene after perturbations [7], [17], [18], [19], [20], [21]. Nevertheless, in [7] the neuromuscular system was found to be predominantly spring-like, in- 


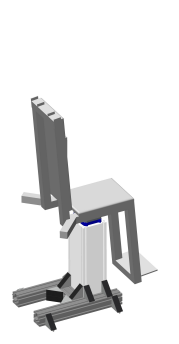

(a) CAD Drawing

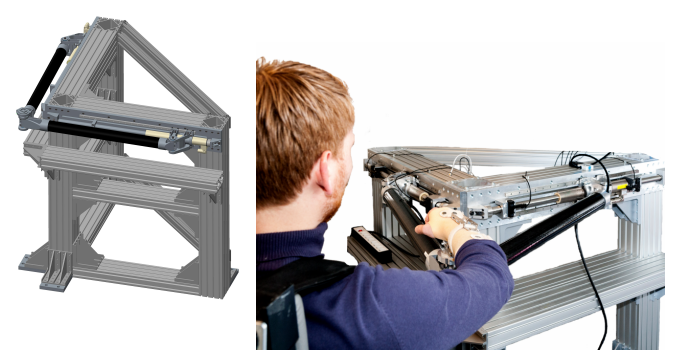

(b) Manipulandum
Fig. 1. Manipulandum

cluding reflexive as well as areflexive responses, but which contradicts the physical definition of stiffness (resistance is provided by elastic/conservative forces only, energy is being stored [22]). Misusing the term stiffness by applying it to arbitrary changes between force and length results in unconventional findings, including negative stiffness [23], [24].

As the described approach generally leads to inconsistencies between identified and modelled stiffness, and in order to develop valuable design guidelines for variablestiffness robots, we argue for in-vivo fast pre-reflexive stiffness measurements. In this paper we describe the development of a new two-dimensional orthoglide mechanism that we primarily developed for revealing purely musculotendon human arm properties over the entire human arm workspace, which we call limb stiffness. The kinematic constraints of the device are shown and first experimental results described.

\section{DESIGN}

Building a robotic manipulandum that can measure intrinsic, pre-reflexive human arm properties inside the entire human arm workspace implies several requirements: (a) the moving mass of the manipulandum needs to be minimal; (b) the structure need to be maximally stiff in order to measure the human and not the manipulandum; (c) the manipulandum must be stronger than the subject; (d) since position perturbations are small displacements with an amplitude around $10 \mathrm{~mm}$, special care needs to be taken to reduce the mechanical clearance.

In order to match these requirements, we took a design decision to not use gears - thus primarily addressing requirements (b) and (d) above - and instead use directdrive actuation based on linear motors.

Along this approach, Wenger et al. [25] investigated the motor arrangement of planar parallel kinematic mechanisms. It was shown that the orthogonal mechanism - introduced by the same authors in [26] and there dubbed the orthoglide mechanism - is better, since it has an optimal quadratic workspace resulting in smaller lengths struts and lower dimensions, better stiffness isotropy, smaller mass in motion, and thus higher dynamic performance [25]. Inspired by this work we decided to build the manipulandum using the orthoglide kinematic arrangement [27].

Knowing that the human elbow joint has an eigenfrequency around $25 \mathrm{~Hz}$ [28], we performed measurements of a perturbation profile with $10 \mathrm{~mm}$ in $10 \mathrm{~ms}$ with a similar type of motor, viz. P01-48 $\times 240-\mathrm{C}$ from NTI AG-LinMot. Based on experiences from experiments on human grip stiffness [29], [30], the test perturbation profile was chosen so as to ensure pre-reflexive measurements for the later human arm case studies. Using that perturbation profile, we found the relevant eigenfrequencies of the motor with different attached masses lying below $62.5 \mathrm{~Hz}$ [27]. Therefore we decided to optimise the eigenfrequency of the structure to be higher than $125 \mathrm{~Hz}$ in order to reduce the influence of the manipulandum eigenfrequency on the human measurements. Using Pro/ENGINEER Mechanica, we simulated different profiles and materials for the structure. We found that only a round profile made of carbon fibre reinforced plastic (CFRP) for each arm is able to match our requirements of a low moving mass with an eigenfrequency above $125 \mathrm{~Hz}$ [27]. Our optimal choice was a CFRP tube of about $70 \mathrm{~mm}$ diameter and $800 \mathrm{~mm}$ length with a flexural stiffness and flexural modulus of elasticity of $6.5 \cdot 10^{10} \mathrm{Nmm}^{2}$ and $230 \mathrm{GPa}$, respectively [27].

A 3D drawing of the device is depicted in Fig. 1. Since the weight of the motors $(2.88 \mathrm{~kg}$ each $)$ is smaller than that of the sliders $(4.12 \mathrm{~kg}$ each), we fixed the sliders to the base instead of the motors. The linear motors P01-48×360 from NTI AG-LinMot provide no guiding functionality. Therefore we use additional ball bearing guides to guide the motors. In order to reduce the mechanical clearance at the end effector, we implemented four parallel bearing guides beside each motor (two on each side, see Fig. 1) instead of one underneath/above the motor. The base is massive, built out of standard aluminium ITEM profiles, and weight approximately $250 \mathrm{~kg}$. At the end effector we added an ATI Mini45 SI-145-5 six-axis force-torque sensor, the orientation of which depends on the motor positions. The motors have a position repeatability of $0.05 \mathrm{~mm}$.

\section{A. Kinematic analysis}

According to [31], the kinematic position equations are formulated by

$$
\begin{aligned}
& L^{2}=\left(p_{x}-\rho_{x}\right)^{2}+p_{y}{ }^{2}, \text { and } \\
& L^{2}={p_{x}}^{2}+\left(p_{y}-\rho_{y}\right)^{2},
\end{aligned}
$$

where $p_{x}$ and $p_{y}$ are the position variables of the end effector, $L=1000 \mathrm{~mm}$ the length of the arms, and $\rho_{x}$ and $\rho_{y}$ the motor positions (please note the difference in the orientations between the coordinate systems of the manipulandum and of the subjects of about $135^{\circ}$; see Fig. 2). Similar to [13] we can derive the differential kinematics by using the Jacobian $\mathbf{J}(\rho)=\left[\delta p_{i} / \delta \rho_{j}\right]$ (find the Jacobian in [31]) and can calculate the condition number over the manipulandum workspace (see Fig. 3(a)). The 


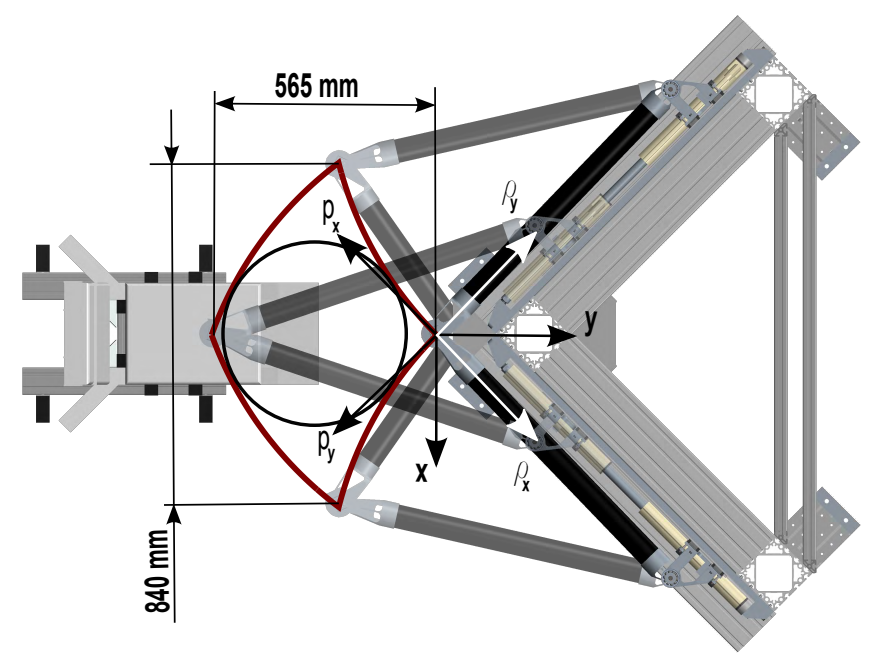

Fig. 2. Workspace and coordinate frames - The reachable workspace and the coordinate frames of the motor $\left[\rho_{x}, \rho_{y}\right]$, of the manipulandum $\left[p_{x}, p_{y}\right]$, and the one used for the performed measurements $[x, y]$ are depicted.

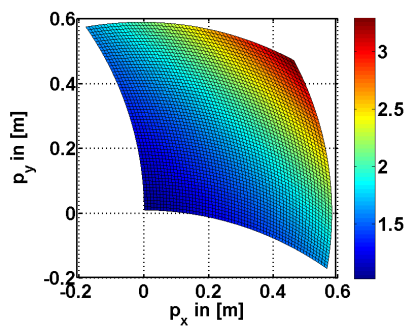

(a) Condition number

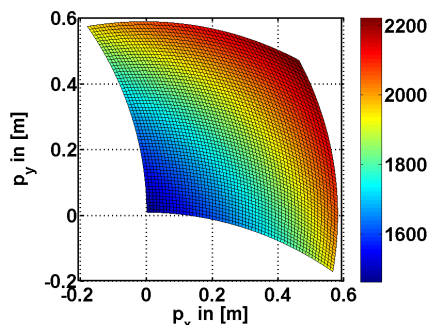

(b) Maximum forces
Fig. 3. Condition number and maximum forces at the end effector- (a) A condition number of the Jacobian of 1 is targeted, meaning an input in motor force or velocity will lead to an equal distributed velocity and force output in end effector space. (b) The norm of the maximum forces which can be achieved at the end effector (note, that the directional data is discarded).

condition number $\operatorname{cond}(\mathbf{J})=\|\mathbf{J}\| \cdot\left\|\mathbf{J}^{-1}\right\|=1 \ldots \infty$ is a quantitative measure of the manipulandum dexterity, where $\|\mathbf{J}\|$ corresponds to the norm of the Jacobian. A condition number of 1 represents the ideal case, meaning that any input force or velocity in motor space results in equally distributed velocities and forces in end effector space between both axes. Fig. 3(a) shows, that for the chosen design condition numbers between 1 and 3.2 can be achieved. Additionally, we used the Jacobian to calculate the norm of maximum end effector forces depending on the workspace by knowing that one motor is able to produce a maximum force of $1024 \mathrm{~N}$ (see Fig. 3(b)).

\section{B. Safety concept}

In order to ensure subject safety, different safety mechanisms working in parallel were developed (see Fig. 4):

- First of all, the arrangement between manipulandum and chair is chosen such that the manipulandum is never able to reach the chest of the subject.

- Secondly, mechanically adjustable stops ensure that the workspace of the manipulandum is never larger than the workspace of the arm of the subject.

- Additionally, there is an emergency stop (ultimate limit switch) at each of the four mechanical stops. If the manipulandum reaches one of the mechanical stops, both motors are switched off instantaneously. When switched off, the manipulandum is always easily backdrivable.

- There are two more emergency stops: one attached to the chair and which can be reached by the subject's left arm, and one for the experimenter. If either one is pressed, both motors are switched off instantaneously.

- The subject's arm is coupled to the manipulandum with a plastic cuff using a permanent magnetic safety clutch in order to allow the subject to decouple in each experimental situation.

- For activating the measurement setup, the subject must hold a dead man's switch with the right foot. If released, no currents are commanded to the motors.

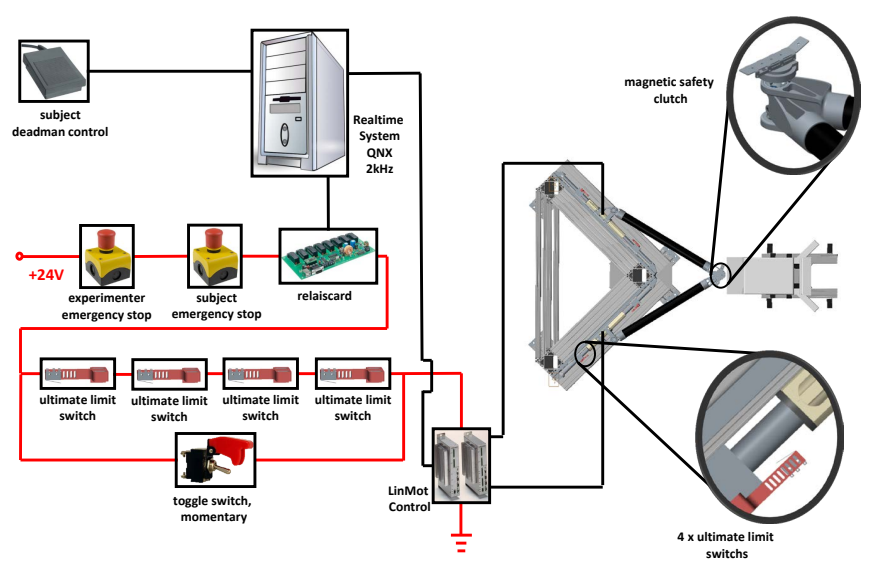

Fig. 4. Safety concept- (a) Four adjustable mechanical stops limit the workspace to the workspace of the subjects; (b) at each of the four mechanical stops emergency stops (ultimate limit switches) are attached; (c) additionally, there is one experimenter and one subject emergency stop; (d) the coupling between subjects arm and manipulandum is realised using a permanent magnetic safety clutch; (e) during the experimental procedure, the subject dead man's switch need to be hold by the subject. The toggle switch is necessary for initialising the motors; if pressed, the ultimate limit switches are bridged.

\section{Measurement setup}

The measurement setup consists of a host running Linux, and a real-time target machine running QNX where a Matlab/Simulink model to control the device is running at $2 \mathrm{kHz}$. The sensors signals are amplified and measured with an analogue-digital converter. The LinMot E1200 motor control boxes are controlled using an Ethercat connection. Both motor control boxes and analogue-digital converter are directly connected to the real-time machine. The nominal range of the ATI force sensor in $x$ and $y$ is $145 \mathrm{~N}$ with a resolution of $1 / 16 \mathrm{~N}$. The nominal range was chosen to allow a proper identification 
with a suitable sensor resolution of changes in forcesand thus optimising the measurement of stiffness - rather than setting it up to measure maximally exertable arm forces.

\section{First EXPERIMENTS}

\section{A. Experiments using an arm dummy}

Initially, we designed an arm dummy based on standard parameters from literature with arm segment lengths [32] of forearm and upper arm of $250 \mathrm{~mm}$ and $330 \mathrm{~mm}$, respectively. We set the maximal subject mass to $100 \mathrm{~kg}$ and designed the segment masses according to [33] of forearm (including the hand) and upper arm to be $2.1 \mathrm{~kg}$ and $2.7 \mathrm{~kg}$, respectively. In order to identify the influence of the quantity of inertia which is wrongly interpreted as subject arm stiffness, we performed two sets of experiments: (a) We attached the arm dummy to the end effector and placed its shoulder at a similar position where the shoulder of the subject will be and performed the experimental protocol below (naturally, no external forces where applied); (b) we repeated the same experiment without the arm dummy.

\section{B. Experimental Procedure}

A total of 5 healthy right-handed male subjects, age 27-35 years, performed the experimental protocol designed to measure their arm stiffness during the application of distal forces measured in one position using the proposed manipulandum (the definition of the distal direction is chosen according to standard literature [7], $[8],[18])$. The experimental procedure is similar to our previous publications, which we call "force task" [29], [30]. All subjects gave written consent to the experimental procedures. Subjects were seated on a special adjustable chair fixed to the base. The subject's chest was restrained with seat belts to limit movement to arms only. The end effector was placed at a central position such that a line between shoulder and end effector would be parallel to the distal direction, so as to reach elbow angles around $90^{\circ}$. The end effector is connected to subjects arm via a plastic cuff; the elbow is assisted by a belt in order to compensate for the weight of the arm. The workspace of the manipulandum and the height of the chair were adapted to comfortably seat the subject. Initially, subjects were asked to apply maximum force in the distal direction (positive y-axis; see Fig. 2), while their maximum voluntary contraction level in that direction was estimated. 3 of 5 subjects reached the force limit of the force sensor $(145 \mathrm{~N})$; in this case $160 \mathrm{~N}$ was chosen arbitrary which lead to stable results without having subjects fatiguing too fast. Subsequently, subjects were shown visual feedback about the applied forces in a plane parallel to the actuator movement using a dot, and a circle representing a required amount of distal force with a tolerance of $2.0 \%$. Subjects were then asked to reach that distal force level with the aid of the presented circle, and keep it until the perturbation was felt. Since no influence is expected from active control and in contrast to the do-not-voluntarily-intervene paradigm, subjects were allowed to relax if perturbation was felt. The required normalised distal force levels were either at $20,30,40,50$, or $60 \%$ of the maximum force and will be referred as NFL1 ..5. The perturbation consists of a $12 \mathrm{~mm}$ displacement (please note that the displacement was interpolated in end effector coordinates rather than motor positions) of the hand in 8 different directions $(0$, $45,90,135,180,225,270$, and $315^{\circ}$; again, please note the difference in orientation between the coordinate systems of the manipulandum and of the subject of about $135^{\circ}$; see Fig. 2). Each combination of normalised distal force level and perturbation direction was repeated 5 times, leading to 200 trials in total per subject which were presented in a randomised order. After each perturbation the hand was moved back to the central position and subjects had to rest for approximately 10 seconds. In between tasks subjects were again asked to relax instead of using the do-not-voluntarily-intervene paradigm. Given a visual feedback we used this relaxation phase for automatically resetting the force sensor (similar to [30] perturbations lead to small drifts in the force signal). If subjects reported fatigue, they would be allowed to rest as much as needed. The whole experiment lasted about 90 minutes per subject; no subject reported discomfort.

\section{Data Processing}

The force signals were first filtered using a 21-point moving average filter. The length of the time window before the perturbation was set to $50 \mathrm{~ms}$, while the time window length after the perturbation was chosen to $100 \mathrm{~ms}$; the last data point for the evaluation of stiffness was taken $200 \mathrm{~ms}$ after the onset of the perturbation (see Fig. 5; see [34] for a more detailed analysis about the influence of choosing time windows on the mechanical response). These time window length were chosen by visual inspection of the respective position, velocity, and acceleration signals under the premiss of having almost no influence of damping and inertial properties. The differences in the mean values over these two time windows were evaluated for force and position and the Cartesian stiffness matrix was determined by L2 regression. Please note that we refrain from measuring arm joint angles and are therefore able to only assess Cartesian stiffness.

To compare the results across subjects and studies we will represent the measured stiffness matrices as ellipses and describe them using the four metrics $K_{\text {size }}, K_{\text {shape }}$, $K_{\text {orient }}$, and $Z_{\text {mean }}$ introduced in [7], [35].

The size of the stiffness ellipse is computed by

$$
K_{\text {size }}=\left|\pi \lambda_{\max } \lambda_{\min }\right|,
$$

which is a quantitative representation of the measured amount of stiffness. $\lambda_{\max / \min }$ are the eigenvalues and denote the stiffness in the direction of the major and minor axis of the stiffness ellipse, respectively. 

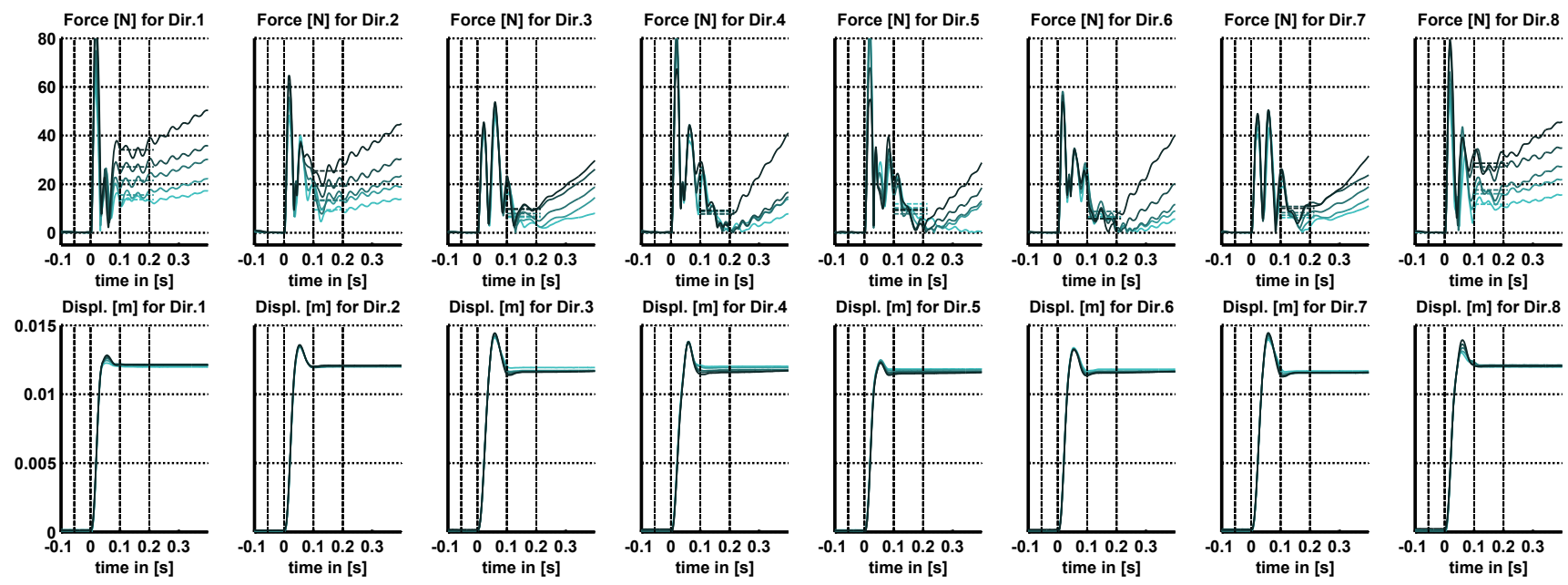

Fig. 5. Exemplary force and displacement profiles (rows) of Subject $\mathbf{S} 4$ for each perturbation direction (columns) and force level (colours) - The mean force (upper row) and mean displacement profiles (lower row) shown corresponds to the norm of the perturbation along the axis of perturbation. Direction 1 corresponds to the distal pushing direction (positive y-axis; see Fig. 2) while the number of perturbation direction increases clockwise. The line colours indicate the NFL, while the darker colours refer to the higher NFL. Note, that the means over the time window before the perturbation were subtracted from each force profile. The start of the perturbation is indicated as $\mathrm{t}=0 \mathrm{~s}$. The time window positions are depicted by 4 vertical dashed lines with the end of the first time window at $\mathrm{t}=0 \mathrm{~s}$. Additionally, the mean force values taken over the second time window are shown as coloured dashed horizontal lines.

The shape of the stiffness ellipse is computed by

$$
K_{\text {shape }}=\left|\frac{\lambda_{\max }}{\lambda_{\min }}\right|
$$

and is a qualitative characterisation of isotropy of the endpoint stiffness [36]. $K_{\text {shape }}$ of $100 \%$ represents an ideal isotropic endpoint stiffness, i.e., the endpoint stiffness can be represented by a circle. It means that a force perturbing the endpoint in any direction would lead to the same proportional restoring force and displacement in the opposite direction [36].

Similar to [7] the orientation of the stiffness ellipses can be calculated using the definition of the dot product in Euclidean space

$$
K_{\text {orient }}=\arccos \left(\left(\begin{array}{c}
-1 \\
0
\end{array}\right) \cdot v\right)
$$

where $v$ denotes the normalized eigenvector corresponding to $\lambda_{\max }$ and $K_{\text {orient }}$ is the angle between the negative $x$-axis and $v[36]$.

Additionally, we use $Z_{\text {mean }}$ which is the square root of the relation between the determinants of asymmetric and symmetric components of the measured stiffness matrix

$$
Z_{\text {mean }}=\sqrt{\frac{\operatorname{det}\left(K_{\mathrm{asym}}\right)}{\operatorname{det}\left(K_{\mathrm{sym}}\right)}},
$$

and which was introduced by Mussa-Ivaldi et al. in 1985 [7]. $Z_{\text {mean }}$ compares the influence of non-spring-like forces on measured stiffness matrices and is independent of the used coordinate frame (joint or Cartesian space). A $Z_{\text {mean }}$ value of $0 \%$ determines an ideal elastic behaviour (perfect symmetric stiffness matrix), while $100 \%$ shows that the measured forces are not originating from a spring-like force field. We will use it to identify the amount of inertia and damping (non-conservative part) which is wrongly identified as stiffness (conservative part; see [7] for details).

Finally, the measured Cartesian stiffness matrix of an ideally isolated system need to be positive definite which we will determine as well.

\section{Results}

The experimental results are represented in Fig. 6 and Table I. Fig. 6 shows the measured Cartesian stiffness matrices represented as ellipses. Additionally, the mean reaction forces and displacements are shown in Fig. 6 for each force level and perturbation direction. Table I summarises the four metrics used to compare the measured stiffness matrices. While we found no significant correlation between $K_{\text {size }}$ or $Z_{\text {mean }}$ and the normalised force level, we found for both $K_{\text {orient }}$ and $K_{\text {shape }}$ a significant correlation $(\mathrm{p} \leq 0.001)$ to normalised force with $r=0.66$ and $r=-0.63$, respectively (Pearson's $r$ ). All measured stiffness matrices were found to be positive definite. Additionally, using $Z_{\text {mean }}$ we found that the quantity of inertia and damping which is erroneously interpreted as stiffness for the manipulandum with and without attached arm dummy in comparison to all identified subject stiffness to be $4.9 \pm 0.79 \%$ and $0.79 \pm 0.13 \%$, respectively. Analogously, we found mean influence of about $6.8 \pm 1.0 \%$ over all force levels and subjects of non-springlike forces on our regressed stiffness with its maximum for the fifth force level of subject S1.

\section{Discussion And Conclusion}

We have introduced a new robotic manipulandum based on an orthoglide mechanism. The goal of this ma- 


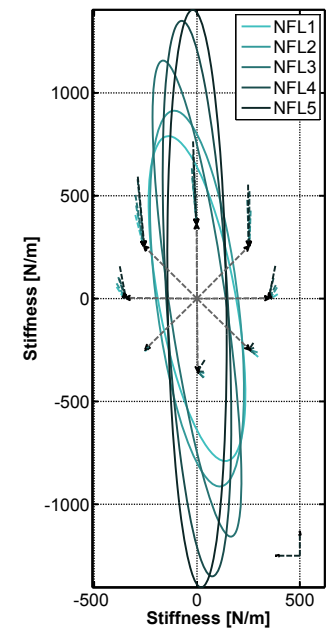

(a) Subject S1

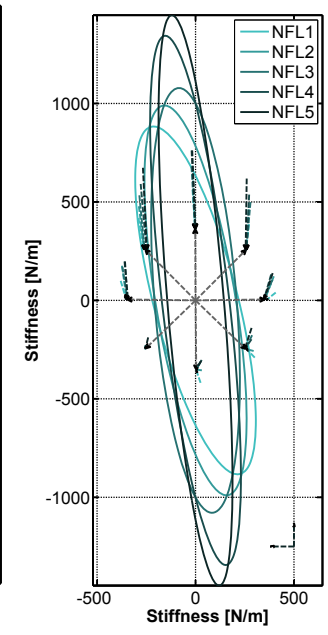

(b) Subject S2

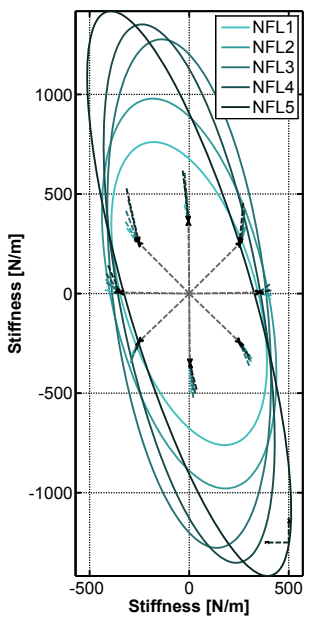

(c) Subject S3

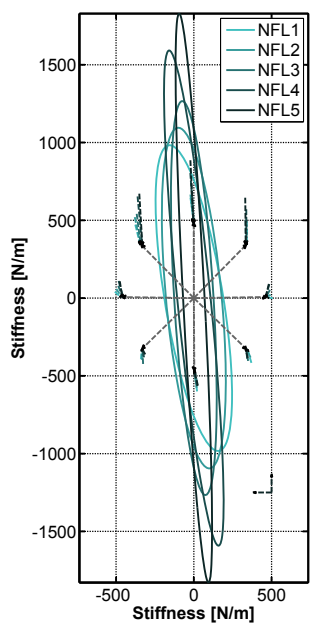

(d) Subject S4

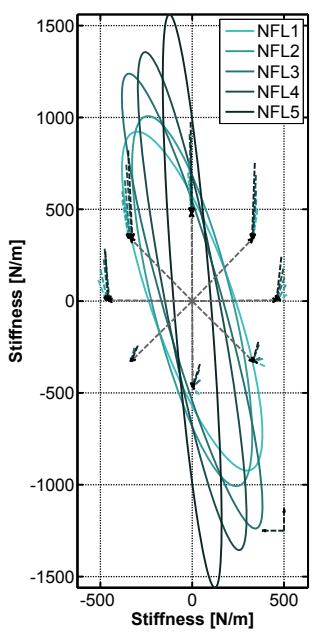

(e) Subject S5

Fig. 6. Experimental results - The figures show the measured Cartesian stiffness matrices represented as ellipses for all subjects and force levels (colours). Additionally, the mean reaction forces and displacements are shown for each force level and perturbation direction. The depicted forces are scaled with the constant position displacements of $0.012 \mathrm{~mm}$; exemplary, the two arrows in the lower right half of the figures corresponds to $10 \mathrm{~N}$ each. The line colours indicate the NFL, while the darker colours refer to the higher NFL.

nipulandum is to identify intrinsic human arm impedance parameters, by performing arm perturbation measurements before fast reflex effects change stiffness and/or damping. Even if we were not able to keep the end of the second time window for estimating stiffness below $50 \mathrm{~ms}$ and thus the onset of measurable fast reflex responses in force, visual inspection of the perturbation profiles in Fig. 5 argues for the successful identification of stiffness before the onset of relevant active control. Furthermore, measurements using the manipulandum with and without an arm dummy shows that less than $5 \%$ of the identified stiffness matrix originates from wrongly interpreted inertia. All identified stiffness matrices were found to be positive definite and the influence of nonconservative components showed to have an influence of approximately $6.8 \pm 1.0 \%$ on our measurements. In comparison, [7] reported a $Z_{\text {mean }}$ less than $21 \%$, corresponding to our finding of less than $20 \%$. Moreover, we found the orientation of the stiffness ellipse increasing and the ellipse shape decreasing with the applied force. In other words, the higher the applied forces, the thinner the measured ellipse, with its major axis turning towards the direction of pushing. Thereby, the orientation and shape changed on average about $5.7 \pm 2.1^{\circ}$ and $16.0 \pm 3.2 \%$. Interestingly and contrary to our initial expectations, we found no relevant trend for the size of the ellipses. Nevertheless, the length of major axis of the stiffness ellipse increases linearly with the applied force $(r=0.93$, $\mathrm{p} \leq 0.0001$ ), while there is no significant correlation for its small eigenvalue.

Furthermore, even for the first force level NFL1 the sizes of the measured stiffness ellipses are substantially larger than those reported in [7] measured in a posture maintaining task. Analysing the differences, we found that position displacements of 5 and $8 \mathrm{~mm}$ were used in [7] in comparison to $12 \mathrm{~mm}$ used in this work. Moreover, the rising times for the perturbations differ considerably. Nevertheless, van Doren reported a decreasing stiffness with an increasing perturbation amplitude and an increasing stiffness with an increasing rising time [37], arguing in the opposite direction for both differences. Furthermore, we asked subjects in our experiment to relax after the perturbation, in contrast with the donot-intervene-voluntarily paradigm, also arguing in the opposite direction. All in all, it is most-likely that the differences found can be attributed to a larger mechanical response of more recruited motor units caused by the comparatively "earlier" identification of stiffness [34].

Notice that the force sensor was chosen so as to measure stiffness as accurately as possible. We selected a sensor with a limited force range; therefore, however, we were not able to measure maximum voluntary contraction. Furthermore, we measured stiffness always at the same reference position, independent of arm subjects kinematics. However, the chosen central position corresponds to an elbow angle between forearm and upper arm above $90^{\circ}$ and lead to more elongated ellipses rather than isotropic ones with a limited change in orientation.

Conclusively, the device will help us understanding how to choose limits for robotic stiffness and relations for a proper cam disc design in variable stiffness actuators. We will further improve the perturbation profile, i.e., reducing the perturbation amplitude to $8 \mathrm{~mm}$ which is used in standard literature [7] in order to reduce the end of the second time window. For future applications, we plan to relate measured stiffness to electromyographic data in order to measure stiffness continuously without the usage of disturbing perturbations. These relations will allow us to control the compliance of robotic systems in tele-operational approaches. 
TABLE I

Experimental results.

\begin{tabular}{c||c|c|c|c} 
Subject & $K_{\text {size }}$ & $K_{\text {orient }}$ & $K_{\text {shape }}$ & $Z_{\text {mean }}$ \\
\hline \hline S1-NFL1 $^{1}$ & $47.3(\mathrm{~N} / \mathrm{cm})^{2}$ & $73.5^{\circ}$ & $23.6 \%$ & $10.2 \%$ \\
S1-NFL2 $^{1}$ & $57.3(\mathrm{~N} / \mathrm{cm})^{2}$ & $74.6^{\circ}$ & $22.0 \%$ & $14.8 \%$ \\
S1-NFL3 $^{1}$ & $50.8(\mathrm{~N} / \mathrm{cm})^{2}$ & $78.6^{\circ}$ & $11.9 \%$ & $8.1 \%$ \\
S1-NFL4 $^{1}$ & $63.3(\mathrm{~N} / \mathrm{cm})^{2}$ & $82.3^{\circ}$ & $11.1 \%$ & $11.7 \%$ \\
S1-NFL5 $^{1}$ & $63.1(\mathrm{~N} / \mathrm{cm})^{2}$ & $82.0^{\circ}$ & $10.3 \%$ & $19.7 \%$ \\
\hline S2-NFL1 $^{1}$ & $61.0(\mathrm{~N} / \mathrm{cm})^{2}$ & $80.1^{\circ}$ & $23.6 \%$ & $7.7 \%$ \\
S2-NFL2 $^{1}$ & $63.9(\mathrm{~N} / \mathrm{cm})^{2}$ & $81.1^{\circ}$ & $20.2 \%$ & $1.0 \%$ \\
S2-NFL3 $^{1}$ & $70.9(\mathrm{~N} / \mathrm{cm})^{2}$ & $82.3^{\circ}$ & $19.3 \%$ & $5.6 \%$ \\
S2-NFL4 $^{1}$ & $72.5(\mathrm{~N} / \mathrm{cm})^{2}$ & $82.9^{\circ}$ & $12.6 \%$ & $1.2 \%$ \\
S2-NFL5 $^{1}$ & $65.6(\mathrm{~N} / \mathrm{cm})^{2}$ & $83.4^{\circ}$ & $9.9 \%$ & $4.5 \%$ \\
\hline S3-NFL1 $^{1}$ & $83.1(\mathrm{~N} / \mathrm{cm})^{2}$ & $75.8^{\circ}$ & $42.8 \%$ & $2.2 \%$ \\
S3-NFL2 $^{1}$ & $122(\mathrm{~N} / \mathrm{cm})^{2}$ & $78.1^{\circ}$ & $39.0 \%$ & $0.8 \%$ \\
S3-NFL3 $^{1}$ & $155(\mathrm{~N} / \mathrm{cm})^{2}$ & $81.5^{\circ}$ & $29.9 \%$ & $2.4 \%$ \\
S3-NFL4 $^{1}$ & $152(\mathrm{~N} / \mathrm{cm})^{2}$ & $81.3^{\circ}$ & $25.7 \%$ & $3.2 \%$ \\
S3-NFL5 $^{1}$ & $146(\mathrm{~N} / \mathrm{cm})^{2}$ & $79.0^{\circ}$ & $21.5 \%$ & $9.5 \%$ \\
\hline S4-NFL1 & $58.7(\mathrm{~N} / \mathrm{cm})^{2}$ & $80.9^{\circ}$ & $18.9 \%$ & $0.5 \%$ \\
S4-NFL2 $^{1}$ & $61.8(\mathrm{~N} / \mathrm{cm})^{2}$ & $81.6^{\circ}$ & $16.3 \%$ & $6.5 \%$ \\
S4-NFL3 $^{1}$ & $50.5(\mathrm{~N} / \mathrm{cm})^{2}$ & $84.4^{\circ}$ & $10.0 \%$ & $6.0 \%$ \\
S4-NFL4 $^{1}$ & $53.8(\mathrm{~N} / \mathrm{cm})^{2}$ & $84.8^{\circ}$ & $6.7 \%$ & $1.7 \%$ \\
S4-NFL5 $^{1}$ & $39.9(\mathrm{~N} / \mathrm{cm})^{2}$ & $85.1^{\circ}$ & $3.8 \%$ & $8.8 \%$ \\
\hline S5-NFL1 $^{1}$ & $68.7(\mathrm{~N} / \mathrm{cm})^{2}$ & $76.6^{\circ}$ & $23.3 \%$ & $9.7 \%$ \\
S5-NFL2 $^{1}$ & $70.3(\mathrm{~N} / \mathrm{cm})^{2}$ & $76.6^{\circ}$ & $20.8 \%$ & $1.1 \%$ \\
S5-NFL3 $^{1}$ & $65.4(\mathrm{~N} / \mathrm{cm})^{2}$ & $82.1^{\circ}$ & $12.8 \%$ & $19.4 \%$ \\
S5-NFL4 $^{1}$ & $63.4(\mathrm{~N} / \mathrm{cm})^{2}$ & $81.2^{\circ}$ & $10.6 \%$ & $5.4 \%$ \\
S5-NFL5 $^{1}$ & $49.9(\mathrm{~N} / \mathrm{cm})^{2}$ & $83.2^{\circ}$ & $6.5 \%$ & $7.7 \%$ \\
1 $^{2}$ & & &
\end{tabular}

1 The stiffness matrix is positive definite.

Representation of the measured stiffness matrices using $K_{\text {size }}$, $K_{\text {shape }}, K_{\text {orient }}$, and $Z_{\text {mean }}$.

\section{ACKNOWLEDGEMENTS}

We want to thank Maximilian Große-Dunker, Jörn Vogel and Constantin Böhm for their assistance in mounting, controlling and designing the manipulandum.

\section{REFERENCES}

[1] A. Albu-Schäffer, S. Haddadin, C. Ott, A. Stemmer, T. Wimböck, and G. Hirzinger. The DLR lightweight robot: design and control concepts for robots in human environments. Industrial Robot: an international journal, 34(5):376-385, 2007.

[2] J.H. Allum and K.H. Mauritz. Compensation for intrinsic muscle stiffness by short-latency reflexes in human triceps surae muscles. Journal of Neurophysiology, 52(5):797-818, 11 1984.

[3] N. Hogan. Adaptive control of mechanical impedance by coactivation of antagonist muscles. Automatic Control, IEEE Transactions on, 29(8):681-690, Aug 1984.

[4] M. Grebenstein, A. Albu-Schäffer, T. Bahls, and et al. The DLR Hand Arm System. In IEEE International Conference on Robotics and Automation (ICRA), pages 3175-3182, May 2011.

[5] B. Vanderborght, A. Albu-Schäffer, A. Bicchi, and et al. Variable impedance actuators: A review. Robotics and Autonomous Systems, 61(12):1601-1614, 2013.

[6] S. Wolf, T. Bahls, M. Chalon, and et al. Soft robotics with variable stiffness actuators: Tough robots for soft human robot interaction. In A. Verl, A. Albu-Schäffer, O. Brock, and A. Raatz, editors, Soft Robotics, pages 231-254. Springer Berlin Heidelberg, 2015.

[7] F.A. Mussa-Ivaldi, N. Hogan, and E. Bizzi. Neural, mechanical, and geometric factors subserving arm posture in humans. Journal of Neuroscience, 5(10):2732, 1985.

[8] E. de Vlugt, A.C. Schouten, F.C.T. van der Helm, P.C. Teerhuis, and G.G. Brouwn. A force-controlled planar haptic device for movement control analysis of the human arm. Journal of Neuroscience Methods, 129(2):151-168, October 2003.
[9] N. Hogan, H.I. Krebs, J. Charnnarong, P. Srikrishna, and A. Sharon. Mit-manus: a workstation for manual therapy and training. i. In Robot and Human Communication, 1992. Proceedings., IEEE International Workshop on, pages 161165 , Sep 1992.

[10] I.S. Howard, J.N. Ingram, and D.M. Wolpert. A modular planar robotic manipulandum with end-point torque control. Journal of Neuroscience Methods, 181(2):199 - 211, 2009.

[11] M. Casadio, V. Sanguineti, P.G. Morasso, and V. Arrichiello. Braccio di ferro: a new haptic workstation for neuromotor rehabilitation. Technology and Health Care, 14(3):123-142, 2006.

[12] H. Gomi and M. Kawato. Equilibrium-point control hypothesis examined by measured arm stiffness during multijoint movement. Science (New York, N.Y.), 272(5258):117-20, 1996.

[13] J. Klein, N. Roach, and E. Burdet. 3DOM: a 3 degree of freedom manipulandum to investigate redundant motor control. IEEE transactions on haptics, 7(2):229-39, 2013.

[14] D. Campolo, P. Tommasino, K. Gamage, J. Klein, C. Hughes, and L. Masia. H-Man: A planar, H-shape cabled differential robotic manipulandum for experiments on human motor control. Journal of neuroscience methods, 235:285-97, September 2014.

[15] L. Masia, V. Squeri, G. Sandini, and P. Morasso. Measuring end-point stiffness by means of a modular mechatronic system. In Robotics and Automation (ICRA), 2012 IEEE International Conference on, pages 2471-2478, May 2012.

[16] M.L. Latash. Virtual trajectories and joint stiffness reconstructed with smooth perturbation technique under different instructions. Journal of Biomechanics, 27(6):732, 1994.

[17] R. Osu, D.W. Franklin, H. Kato, H. Gomi, K. Domen, T. Yoshioka, and M. Kawato. Short- and long-term changes in joint co-contraction associated with motor learning as revealed from surface EMG. Journal of neurophysiology, 88(2):991-1004, 2002.

[18] R. Osu and H. Gomi. Multijoint muscle regulation mechanisms examined by measured human arm stiffness and emg signals. pages 1458-1468, 2005.

[19] J. McIntyre, F.A. Mussa-Ivaldi, and E. Bizzi. The control of stable postures in the multijoint arm. Experimental brain research, 110(2):248-64, July 1996.

[20] H. Gomi and M. Kawato. Human arm stiffness and equilibrium-point trajectory during multi-joint movement. $B i$ ological cybernetics, 76(3):163-171, 1997.

[21] R. Shadmehr, F.A. Mussa-Ivaldi, and E. Bizzi. Postural force fields of the human arm and their role in generating multijoint movements. The Journal of neuroscience, 13(1):45-62, 1993.

[22] Mark L. Latash and Vladimir M. Zatsiorsky. Joint stiffness: Myth or reality? Human movement science, 12(6):653-692, 1993.

[23] P. Dyhre-Poulsen, E.B. Simonsen, and M. Voigt. Dynamic control of muscle stiffness and H-reflex modulation during hopping and jumping in man. The Journal of Physiology, 437(1):287-304, 1991.

[24] M.S. Erden and A. Billard. Hand impedance measurements during interactive manual welding with a robot. Robotics, IEEE Transactions on, 31(1):168-179, Feb 2015.

[25] P. Wenger, C. Gosselin, and D. Chablat. A comparative study of parallel kinematic architectures for machining applications. CoRR, abs/0707.3665, 2007.

[26] D. Chablat, P. Wenger, and J. Angeles. Conception isotropique d'une morphologie parallèle: application à l'usinage, 3rd int. In 3th International Conference on Integrated Design and Manufacturing in Mechanical Engineering, May 2000.

[27] C. Böhm. Konzeptionierung und Auslegung eines planaren Manipulators zur reflexfreien Steifigkeitsmessung des menschlichen Arms. Diploma Thesis, DLR interner Bericht, University of Applied Sciences, Munich, April 2013.

[28] Yangming Xu, I.W. Hunter, J.M. Hollerbach, and D.J. Bennett. An airjet actuator system for identification of the human arm joint mechanical properties. Biomedical Engineering, IEEE Transactions on, 38(11):1111-1122, Nov 1991. 
[29] H. Höppner, D. Lakatos, H. Urbanek, Claudio Castellini, and P. van der Smagt. The Grasp Perturbator: Calibrating human grasp stiffness during a graded force task. In Proc. ICRAInternational Conference on Robotics and Automation, pages 3312-3316, 2011.

[30] H. Höppner, J. McIntyre, and P. van der Smagt. Task dependency of grip stiffness - A study of human grip force and grip stiffness dependency during two different tasks with same grip forces. PLoS ONE, 8(12):e80889, 122013.

[31] A. Pashkevich, D. Chablat, and P. Wenger. Kinematics and workspace analysis of a three-axis parallel manipulator: The orthoglide. Robotica, 24(1):39-49, January 2006.

[32] P. de Leva. Adjustments to zatsiorsky-seluyanov's segment inertia parameters. Journal of Biomechanics, 29(9):1223 1230, 1996.

[33] L. Chugunova V. Zatsiorsky, V. Seluyanov. In vivo body segment inertial parameters determination using a gammascanner method. Biomechanics of Human Movement: Applications in Rehabilitation, Sports and Ergonomics, pages 186 202, 1990.

[34] T. R. Nichols and J. C. Houk. Improvement in linearity and regulation of stiffness that results from actions of stretch reflex. Journal of Neurophysiology, 39(1):119-142, 1976.

[35] N. Hogan. The mechanics of multi-joint posture and movement control. Biological Cybernetics, 331:315-331, 1985.

[36] H. Höppner, W. Wiedmeyer, and P. van der Smagt. A new biarticular joint mechanism to extend stiffness ranges. In Robotics and Automation (ICRA), 2014 IEEE International Conference on, pages 3403-3410, May 2014.

[37] C.L. Van Doren. Grasp stiffness as a function of grasp force and finger span. Motor Control, 2(4):352-378, 1998. 